\title{
Carrier mobility characteristics in GalnNAs dilute nitride films grown by atomic hydrogen-assisted molecular beam epitaxy
}

\author{
Naoya Miyashita, ${ }^{\text {a) }}$ Yukiko Shimizu, and Yoshitaka Okada \\ Institute of Applied Physics, University of Tsukuba, 1-1-1 Tennodai, Tsukuba, Ibaraki 305-8573, Japan
}

(Received 9 January 2007; accepted 6 July 2007; published online 23 August 2007)

\begin{abstract}
We have investigated the electrical properties of GaInNAs dilute nitride films grown by atomic hydrogen-assisted molecular beam epitaxy. We found that although the hole mobilities in Be-doped $p$-GaInNAs films exhibit a temperature dependence nearly identical to that for the homoepitaxial $p$-GaAs films, the electron mobilities in Si-doped $n$-GaInNAs films are strongly affected by the introduction of nitrogen into $\mathrm{Ga}(\mathrm{In}) \mathrm{As}$. Further, the degree of scattering by the ionized impurity-like centers generated by $\mathrm{N}$ atoms decreased with increasing Si doping, while neutral impurity-like scattering became more dominant with increasing Si doping. These results suggest that the decrease of electron mobility and carrier concentration in Si-doped $n$-GaInNAs films is strongly correlated with the presence of $\mathrm{N}$ and $\mathrm{Si}$ atoms. (C) 2007 American Institute of Physics.
\end{abstract}

[DOI: $10.1063 / 1.2770833]$

\section{INTRODUCTION}

Recently, III-V-N dilute nitride semiconductors such as GaInNAs with an energy band gap of $\sim 1 \mathrm{eV}$ have become of particular importance for future use in lattice-matched GaInP/GaAs/GaInNAs/Ge 4-junction tandem solar cells, which can achieve solar energy conversion efficiencies of $>40 \%{ }^{1,2}$ However, both the optical and electrical properties of GaInNAs thin films, in general, become increasingly degraded with increasing nitrogen composition. ${ }^{1,3}$

The fundamental mechanism of degradation is still not fully understood, although nitrogen-related deep-level defects are known to be responsible for the reduction of minority carrier lifetime and hence the diffusion length in solar cell structures. ${ }^{3,4}$ Even for low concentrations of $\mathrm{N} \leq 2 \%$, the incorporation of nitrogen, a large electronegative anion, results in a strong perturbation of the conduction-band structure, and the electrical properties are affected by the localized electronic states associated with single $\mathrm{N}$ impurities as well as $\mathrm{N}$ aggregates such as N-N pairs and higher-order clusters. ${ }^{5-7}$ As a result, photocurrents generated in GaInNAs subcells at present are not sufficient for practical use in 4-junction tandem solar cells. ${ }^{8}$

Several groups reported that alloy scattering, induced by a random distribution of $\mathrm{N}$ atoms, is one of the main mechanisms affecting the minority carrier diffusion length. ${ }^{9}$ Kurtz et al. argued that the carrier transport in GaInNAs is limited by large-scale inhomogeneities, similar to the behavior observed in polycrystalline $\mathrm{Si}^{10}$ Recently, it was also shown that In and $\mathrm{N}$ atoms play a notable role in the electron mobility in GaInNAs grown by molecular beam epitaxy (MBE), for which the N-related scattering centers were reduced and carrier mobilities were recovered in the presence of In atoms. $^{11}$

In this work, we investigate the carrier mobility and carrier concentration characteristics as a function of tempera-

\footnotetext{
${ }^{a)}$ Author to whom correspondence should be addressed. FAX: +81-29-8536902; bk200101492@s.bk.tsukuba.ac.jp
}

ture. The GaInNAs films were grown by atomic hydrogenassisted molecular beam epitaxy (H-MBE) with a radiofrequency (rf) nitrogen plasma source. ${ }^{12,13}$ We found that although the hole mobilities in Be-doped $p$-GaInNAs films exhibit a temperature dependence that is nearly identical to that for the homoepitaxial $p$-GaAs films doped with $\mathrm{Be}$, the electron mobilities in Si-doped $n$-GaInNAs films are strongly correlated with the presence of $\mathrm{N}$ and $\mathrm{Si}$ atoms.

\section{EXPERIMENT}

All samples were grown on semi-insulating GaAs (001) substrates by atomic $\mathrm{H}$-assisted rf-MBE. ${ }^{12,13} \mathrm{Si}$ and $\mathrm{Be}$, both of which were standard MBE-grade solid sources, were used as $n$-type and $p$-type dopants, respectively, and the carrier concentration at $300 \mathrm{~K}$ was varied from $2 \times 10^{16}$ to 1 $\times 10^{18} \mathrm{~cm}^{-3}$. The GaInNAs layers of $1 \mu \mathrm{m}$ thickness were grown at a fixed growth temperature of $480{ }^{\circ} \mathrm{C}$. The reactive nitrogen flux was generated by using a rf plasma source, and the $\mathrm{N}$ composition was fixed at $\sim 1.0 \%$, which was confirmed with high-resolution x-ray diffraction. No postgrowth anneal was performed. The Hall measurement was then performed under a standard van der Pauw configuration, and the temperature dependence was recorded over a range between 90 and $500 \mathrm{~K}$. The photoluminescence (PL) was also measured by using a $532 \mathrm{~nm}$ second-harmonic line of an $\mathrm{Nd}: \mathrm{VO}_{4}$ diode-pumped laser, a $250 \mathrm{~mm}$ monochromator, and a liquid-nitrogen cooled InGaAs photodetector.

\section{RESULTS AND DISCUSSION}

Figure 1 shows the room-temperature carrier mobility characteristics measured for as-grown $\mathrm{Ga}_{1-x} \mathrm{In}_{x} \mathrm{~N}_{0.01} \mathrm{As}_{0.99}$ $(x=0.03$ and 0.06$)$ and homoepitaxial GaAs films grown by H-MBE technique with different Be and Si doping concentrations. The background doping concentration in an undoped GaInNAs sample grown by our H-MBE technique was typically below the Hall measurement detection limit of $\sim 10^{15} \mathrm{~cm}^{-3}$, so that $n$ - and $p$-type doping have been accu- 


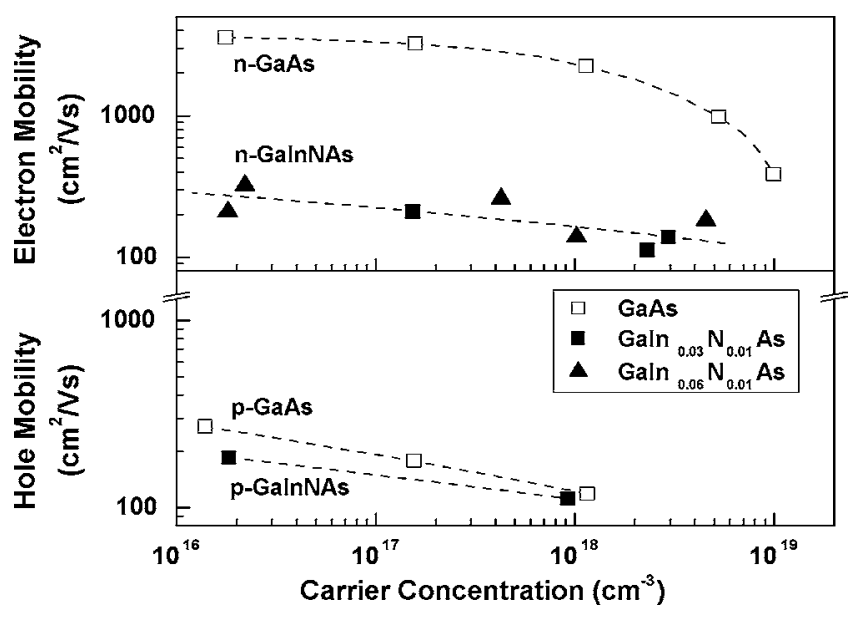

FIG. 1. Room-temperature carrier mobilities measured for Si-doped and Be-doped GaAs and $\mathrm{Ga}_{1-x} \mathrm{In}_{x} \mathrm{~N}_{0.01} \mathrm{As}_{0.99}(x=0.03$ and 0.06$)$ films grown by $\mathrm{H}-\mathrm{MBE}$, plotted as a function of carrier concentration.

rately controlled in our growth system. Further, the secondary ion mass spectroscopy (SIMS) measurements performed in separate experiments revealed that the residual oxygen and carbon impurities in GaInNAs films were below the detection limit of $\sim 10^{17}$ and $10^{16} \mathrm{~cm}^{-3}$, respectively. It can be observed that the majority carrier mobilities in $n$ - and $p$-GaInNAs films were almost comparable, which were $100-300 \mathrm{~cm}^{2} / \mathrm{Vs}$ in $n$-GaInNAs and $100-200 \mathrm{~cm}^{2} / \mathrm{V} \mathrm{s}$ in $p$-GaInNAs films, respectively. Compared to GaAs samples, the hole mobilities in $p$-GaInNAs were insensitive to the introduction of nitrogen. However, the electron mobilities in $n$-GaInNAs films were notably lower by a factor of $\sim 10$. These results suggest that the nitrogen in GaInNAs mainly affects the electron mobilities and not the holes. Further, changing the In composition showed a negligible effect on the electron mobility in $n$-GaInNAs at least for a relatively low composition range of $\leq 6 \%$, as also can be seen from Fig. 1.

Figure 2(a) shows the temperature dependence of electron mobilities measured for $n$ - $\mathrm{Ga}_{1-x} \mathrm{In}_{x} \mathrm{~N}_{0.01} \mathrm{As}_{0.99}(x=0.03$ and 0.06$)$ and $n$-GaAs films with different Si doping concentrations. The electron concentrations measured at $300 \mathrm{~K}$ are also quoted in the figure. Although the carrier mobilities measured for $n$-GaAs exhibited a well-established temperature dependence, the characteristics obtained for $n$-GaInNAs were rather anomalous, reaching maximum values at around $350 \mathrm{~K}$. In particular, the electron mobilities for $n$-GaInNAs samples with carrier concentrations of $2 \times 10^{16}$ and 2 $\times 10^{17} \mathrm{~cm}^{-3}$ at $300 \mathrm{~K}$, which we distinguish as lightly Sidoped $n$-GaInNAs, rapidly decreased with decreasing temperature. The values remained almost constant over a wide temperature range in the heavily Si-doped $\left(4 \times 10^{17}\right.$ and 1 $\times 10^{18} \mathrm{~cm}^{-3}$ ) samples. However, the hole mobilities in $p$ - $\mathrm{Ga}_{0.97} \mathrm{In}_{0.03} \mathrm{~N}_{0.01} \mathrm{As}_{0.99}$ films, as plotted in Fig. 2(b), showed a nearly identical temperature dependence to that for $p$-GaAs films.

It has been an issue of intense discussion that the electron mobilities in $n$-GaInNAs films would become considerably lower than in GaAs due to the conduction-band fluctuations, and $\mathrm{N}$-induced scattering centers and defect states

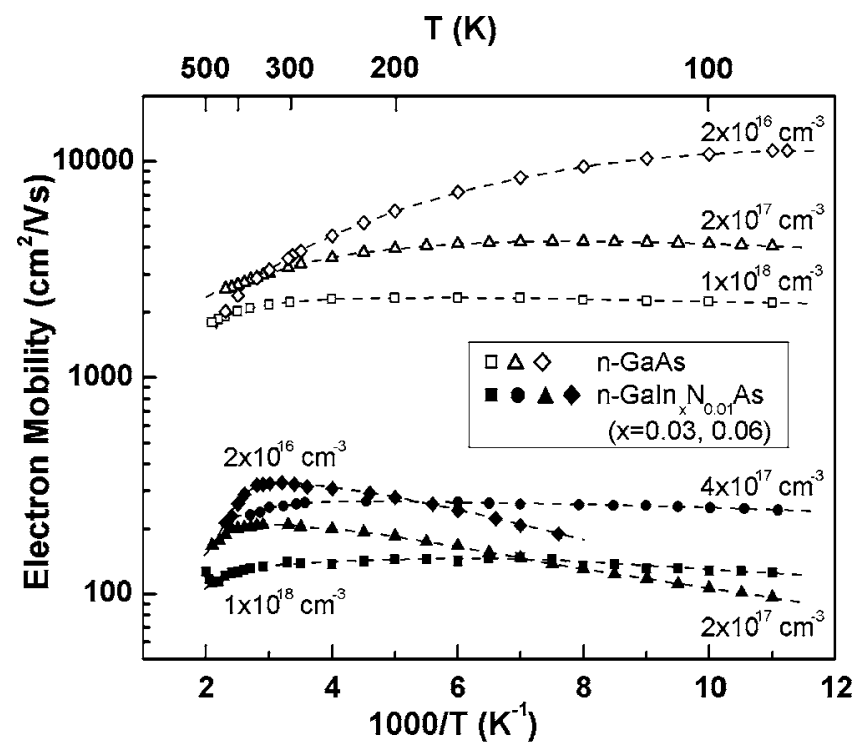

(a)

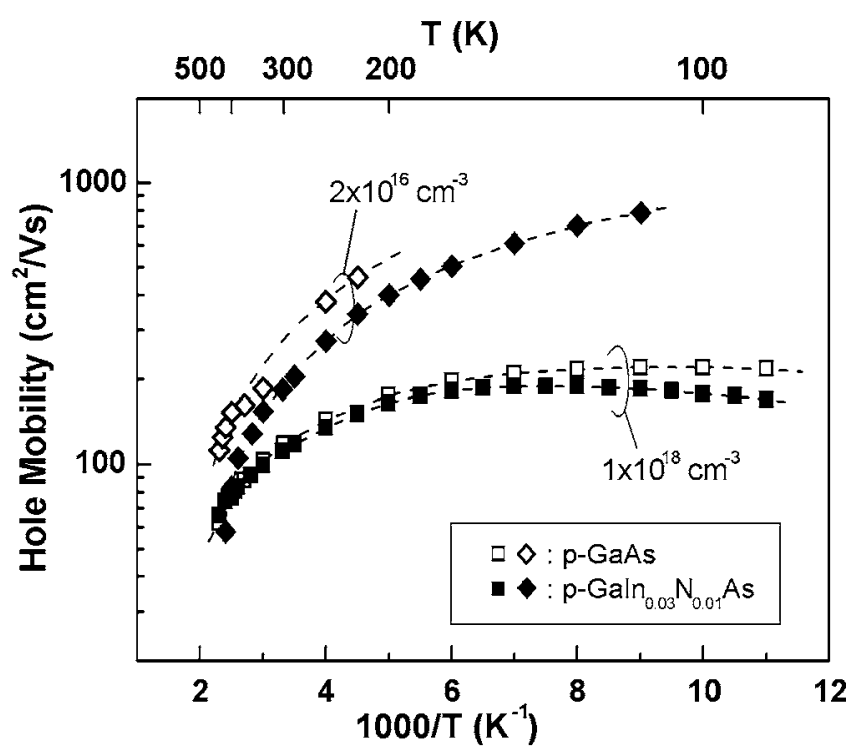

(b)

FIG. 2. Temperature dependence of (a) electron and (b) hole mobilities measured for GaAs (open symbols) and GaInNAs (closed symbols) films grown by H-MBE. The carrier concentrations at room-temperature are also quoted. The dashed lines are just guides to the eye.

formed close to the conduction-band edge of $\mathrm{Ga}(\mathrm{In})$ As. ${ }^{10,11,14,15}$ Therefore, in the lightly doped $n$-GaInNAs films, the electron mobilities are expected to be strongly affected by these scattering centers and defects, and hence decrease rapidly with decreasing temperature. However, this effect would be weakened in the heavily doped $n$-GaInNAs, in which the electron populations in the conduction band are one to two orders of magnitude higher, and hence the potential fluctuations or Coulomb potentials would be screened. As a result, the electron mobilities remain almost constant even at low temperatures. On the contrary, the hole mobilities in $p$-GaInNAs films would be insensitive to the presence of nitrogen.

Next, we analyzed the temperature dependence of carrier mobilities by a standard parameter-fitting process. Here the 
fundamental scattering mechanisms ${ }^{15,16}$ with typical temperature dependence were assumed: $\propto T^{3 / 2}$ (ionized impurity scattering), $\propto T^{-3 / 2}$ (polar optical phonon and deformation potential scattering ${ }^{17}$ ), and $\propto T^{-1 / 2}$ (space-charge scattering ${ }^{18}$ and alloy scattering ${ }^{9}$ ). If each scattering mechanism contributes simultaneously and independently, then the resultant carrier mobility can be given by the Mattiessen's rule,

$$
\mu=\left(\sum_{i} \frac{1}{\mu_{i}}\right)^{-1}
$$

where $\mu_{i}$ is the mobility limited by the $i$ th scattering mechanism. Each $\mu_{i}$ can be written as

$$
\mu_{i}=C_{i} T^{\alpha},
$$

where $C_{i}$ is a constant of the $i$ th scattering mechanism. Reasonably accurate fitting for the lightly Si-doped $n$-GaInNAs $\left(n=2 \times 10^{16}\right.$ and $2 \times 10^{17} \mathrm{~cm}^{-3}$ at $\left.300 \mathrm{~K}\right)$ and Be-doped $p$-GaInNAs samples was obtained by using the above three scattering contributions, i.e., $\propto T^{3 / 2}, \propto T^{-3 / 2}$, and $\propto T^{-1 / 2}$. The electron mobility in $n$-GaInNAs Si-doped to $2 \times 10^{17} \mathrm{~cm}^{-3}$ at $300 \mathrm{~K}$ followed a clear $T^{3 / 2}$ dependence at low temperatures, as shown in Fig. 3(a), thereby indicating a relatively large effect of ionized impurity scattering. However, in the heavily Si-doped samples $\left(n=4 \times 10^{17}\right.$ and $1 \times 10^{18} \mathrm{~cm}^{-3}$ at $300 \mathrm{~K}$ ), accurate fitting curves were not obtained by summation over the above three contributions. A dashed line $\mu_{1}$ in Fig. 3(b) was the best possible fit for the sample with $n=4$ $\times 10^{17} \mathrm{~cm}^{-3}$. It can be seen that one needs to consider additional scattering mechanisms, which result in a weak, if any, temperature dependence of electron mobility. For this, we were able to obtain better results, in both the lightly and heavily Si-doped GaInNAs films, by adding an additional component $\propto T^{0}$. The final results are plotted as solid lines for Fig. 3(a) as well as the curve $\mu_{2}$ in Fig. 3(b).

The temperature-independent scattering mechanism is typically assigned as a neutral impurity scattering. ${ }^{19}$ It is typically observed at relatively low temperatures $\leqslant 100 \mathrm{~K}$, when almost all the shallow donors and acceptors are thermally frozen. In $\mathrm{Ga}(\mathrm{In}) \mathrm{NAs}$ material, it is reported that single $\mathrm{N}$ atoms as well as $\mathrm{N}$ aggregates such as $\mathrm{N}-\mathrm{N}$ pairs and higher-order clusters exhibit neutral impurity-like behavior whose magnitude increases with increasing Si doping. ${ }^{7}$ In order to investigate the effect of neutral impurity-like mobility, $\mu_{\mathrm{NI}}$, we used Erginsoy's result, ${ }^{19}$

$$
\mu_{\mathrm{NI}}=\frac{e}{20 a_{B} \hbar} \frac{m_{e}^{*} / m_{0}}{\varepsilon N_{\mathrm{NI}}}
$$

where $N_{\mathrm{NI}}$ is the neutral impurity concentration, and $\alpha_{B}, \hbar$, and $e$ are the Bohr radius in a hydrogen atom, Planck's constant, and the elementary charge, respectively. As for the dielectric constant $\varepsilon$, we assumed that of GaAs, and $m_{e}^{*} / m_{0}$ $=0.093$ for the effective mass of electrons in GaInNAs containing $1 \%$ nitrogen. ${ }^{5}$ From Fig. $3, \mu_{\mathrm{NI}}=600 \mathrm{~cm}^{2} / \mathrm{V} \mathrm{s}$ for $n=2 \times 10^{17} \mathrm{~cm}^{-3}$ and $500 \mathrm{~cm}^{2} / \mathrm{V} \mathrm{s}$ for $n=4 \times 10^{17} \mathrm{~cm}^{-3}$ were obtained, respectively, from which $N_{\mathrm{NI}}$ was calculated to be $2.0 \times 10^{17}$ and $2.4 \times 10^{17} \mathrm{~cm}^{-3}$, respectively. These values were very close to the Si-doping concentrations and not necessarily the free-electron concentrations at $300 \mathrm{~K}$, and

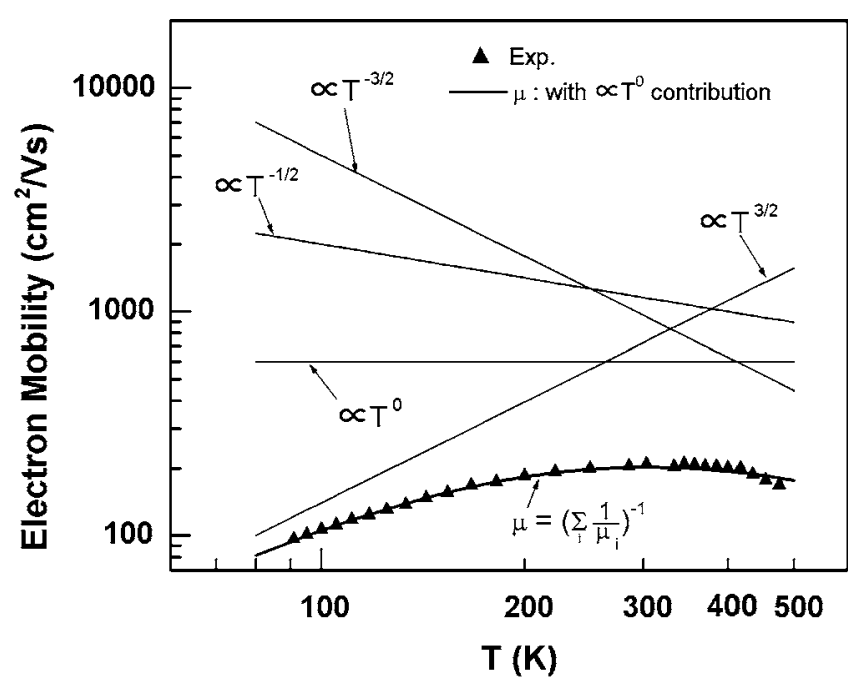

(a)

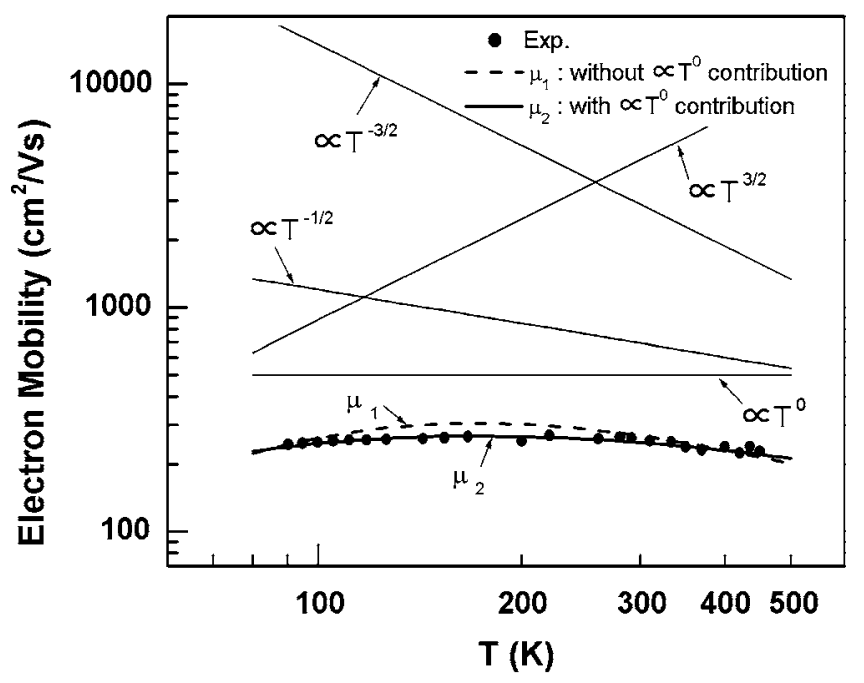

(b)

FIG. 3. Results of parameter fitting on the dependence of electron mobilities on temperature for $n$-GaInNAs films with (a) $n=2 \times 10^{17} \mathrm{~cm}^{-3}$ and (b) $n$ $=4 \times 10^{17} \mathrm{~cm}^{-3}$, respectively. The measured data are plotted as closed triangles in (a) and circles in (b), respectively. Refer to the text for the fitting parameters.

further to be noted was that $N_{\mathrm{NI}}$ increased with $\mathrm{Si}$ doping. The results suggest that the degradation of electron mobilities and carrier concentrations in Si-doped $n$-GaInNAs films is strongly correlated with the presence of $\mathrm{N}$ and $\mathrm{Si}$ atoms, which may exist in the form of Si-N complexes. ${ }^{20-22}$ Other nitrogen-related defects such as N-N (Refs. 5-7) and $\mathrm{N}-\mathrm{H}-\mathrm{V}_{\mathrm{Ga}}$ (Ref. 23) complexes may also be present, but the details are not known at present.

The free-electron concentrations for the $n$-type GaAs and GaInNAs samples measured at different temperatures over a range of $90-500 \mathrm{~K}$ are plotted in Fig. 4. In the heavily Si-doped $n$-GaInNAs samples of $n=4 \times 10^{17}$ and 1 $\times 10^{18} \mathrm{~cm}^{-3}$ at $300 \mathrm{~K}$, the electron concentrations remained almost constant below $300 \mathrm{~K}$. However, in the lightly Sidoped samples of $n=2 \times 10^{16}$ and $2 \times 10^{17} \mathrm{~cm}^{-3}$, the electron concentrations dropped with decreasing temperature. For example, in $n$-GaInNAs with $n=2 \times 10^{17} \mathrm{~cm}^{-3}$, the electron concentration decreased to $3 \times 10^{16} \mathrm{~cm}^{-3}$ at $\sim 90 \mathrm{~K}$. 


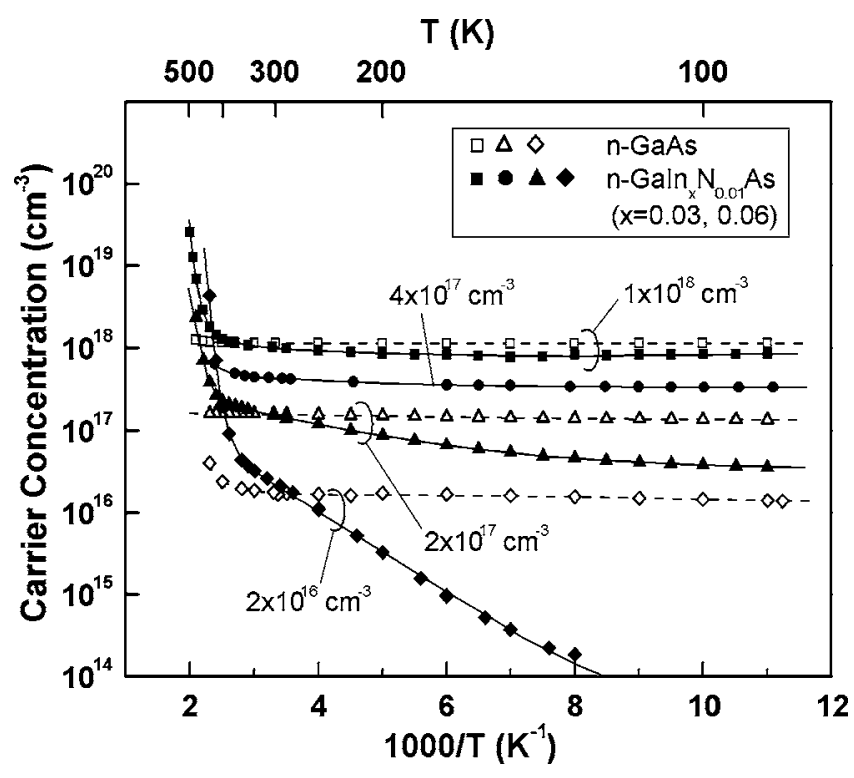

FIG. 4. Temperature dependence of electron concentrations measured for $n$-GaAs (open symbols) and $n$-GaInNAs (closed symbols), respectively. Quoted values are carrier concentrations measured at $300 \mathrm{~K}$. The solid and dashed lines are just guides to the eye.

Since we can safely assume that all the shallow impurity levels were fully ionized at $300 \mathrm{~K}$, the difference of $\sim 1.7$ $\times 10^{17} \mathrm{~cm}^{-3}$ would give an approximate value of the net trap density in this sample. Then the observed dependence of free-carrier concentration for $>100 \mathrm{~K}$ was due to thermal activation of transition between these trap states of energy $E_{T}$ and conduction-band edge $E_{C}$. We determined more precisely the trap densities $N_{T}$ and activation energies given by $E_{a}=E_{C}-E_{T}$ by using the following:

$$
n=\frac{1}{\sqrt{2}}\left(N_{T} N_{C}\right)^{1 / 2} \exp \left(-\frac{E_{a}}{2 k_{B} T}\right)+N_{D}
$$

where the first term is a widely known expression for the electron density in the $n$-type semiconductor and $N_{C}$ is the effective density of states in the conduction band. The second term $N_{D}$ is the ionized donor density, which can be assumed constant since the electron concentration in the $n$-GaAs sample was constant over the temperature range of $\sim 100-400 \mathrm{~K}$. Equation (4) is valid when $N_{D} \gg N_{A}$, the acceptor density, and for the temperature range studied in this work. Fitted results for the $n$-GaInNAs samples with $n=2$ $\times 10^{17}$ and $4 \times 10^{17} \mathrm{~cm}^{-3}$ are shown in Fig. 5. We found that the obtained value of $N_{T}=2 \times 10^{17} \mathrm{~cm}^{-3}$ agreed well with the $N_{\mathrm{NI}}$ value determined by using Eq. (3). Furthermore, the temperature dependence of integrated PL intensity performed in separate experiments showed emission from the localized states at $\sim 70 \mathrm{meV}$ below the conduction-band edge $\left(E_{g}\right.$ $=1.20-1.22 \mathrm{eV}$, at $300 \mathrm{~K})$. This was also consistent with the obtained values of $E_{a}=40-50 \mathrm{meV}$ from Fig. 5. Further, Kurtz et $a{ }^{10}{ }^{10}$ reported $E_{a}=42 \mathrm{meV}$ for Sn-doped $n$-GaInNAs $\left(n=2 \times 10^{16} \mathrm{~cm}^{-3}\right)$, and also calculated that deep donor states formed by $\mathrm{Si}-\mathrm{N}$ nearest-neighbor bonds exist at $230 \mathrm{meV}$ below the GaAs conduction-band minimum. $^{20}$

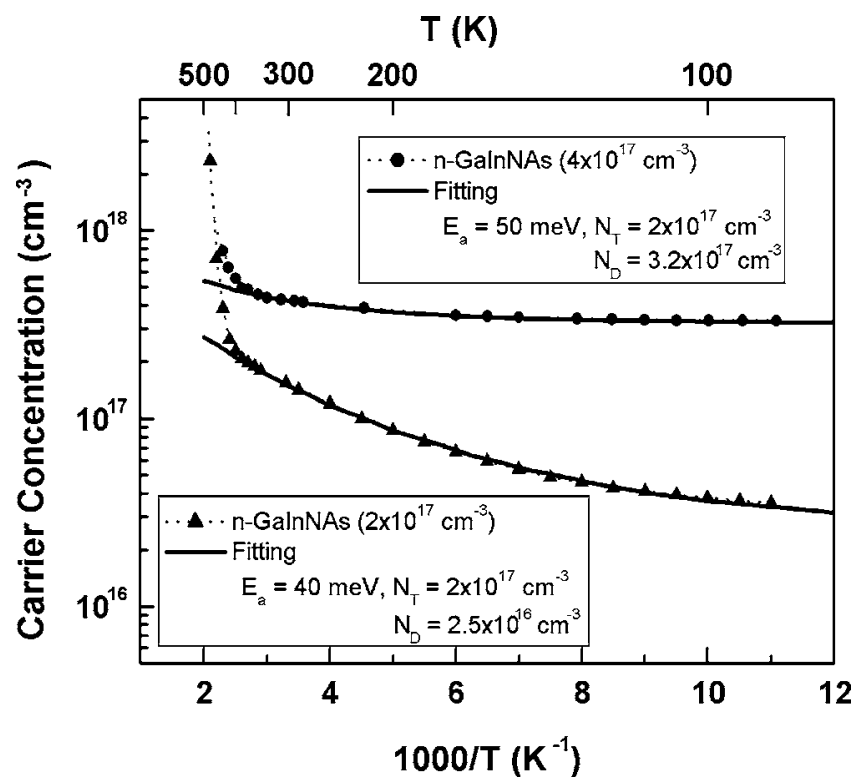

FIG. 5. Results of parameter fitting on the temperature dependence of electron concentrations for $n$-GaInNAs films with (a) $n=2 \times 10^{17} \mathrm{~cm}^{-3}$ and (b) $n=4 \times 10^{17} \mathrm{~cm}^{-3}$, respectively. The measured data are plotted as closed triangles in (a) and circles in (b), respectively.

In this work, it has become clear that not all the $\mathrm{Si}$ impurities are activated to generate free carriers in GaInNAs material. This can be indirectly explained by the coexistence of Si-N complexes, which are known to decrease both the electron concentration and electron mobility. ${ }^{20-22}$ Further, we have shown that these defects may be the origin of neutral impurity scattering that affects mainly the electron mobility. Carrier concentration and mobility in $p$-type GaInNAs doped with $\mathrm{Be}$ were insensitive to nitrogen.

\section{CONCLUSION}

In conclusion, the carrier mobilities and concentrations in $\mathrm{Si}$ - and Be-doped $\mathrm{GaIn}_{x} \mathrm{~N}_{0.01}$ As films grown by H-MBE were investigated. We showed that the effect of ionized impurity-like scattering decreased with increasing Si doping, while the effect of neutral impurity-like scattering became more dominant with increasing Si doping. This could be explained by the formation of $\mathrm{Si}-\mathrm{N}$ complexes that act as neutral impurity scattering centers, thereby affecting the electron mobility and concentration. Other N-related defects such as $\mathrm{N}-\mathrm{N}$ and $\mathrm{N}-\mathrm{H}-\mathrm{V}_{\mathrm{Ga}}$ complexes may also be responsible, and further investigation is necessary to clarify their effects on the electronic properties. Carrier concentration and mobility in $p$-type GaInNAs doped with Be were insensitive to nitrogen.

\section{ACKNOWLEDGMENTS}

This work was supported by the Incorporated Administrative Agency New Energy and Industrial Technology Development Organization (NEDO) under the Ministry of Economy, Trade and Industry (METI), Japan. We also thank Professor K. Akimoto for technical assistance with the Hall measurements.

${ }^{1}$ D. J. Friedman, J. F. Geisz, S. R. Kurtz, and J. M. Olson, J. Cryst. Growth 
195, 409 (1998)

${ }^{2}$ M. Yamaguchi, Physica E (Amsterdam) 14, 84 (2002).

${ }^{3}$ A. M. Mintairov, T. H. Kosel, J. L. Merz, P. A. Blagnov, A. S. Vlasov, V. M. Ustinov, and R. E. Cook, Phys. Rev. Lett. 87, 277401 (2001).

${ }^{4}$ J.-M. Chauveau, A. Trampert, K. H. Ploog, and E. Tournié, Appl. Phys. Lett. 84, 2503 (2004).

${ }^{5}$ S. B. Zhang and S.-H. Wei, Phys. Rev. Lett. 86, 1789 (2001).

${ }^{6}$ M. Reason, H. A. Mckay, W. Ye, S. Hanson, and R. S. Goldman, Appl. Phys. Lett. 85, 1692 (2004).

${ }^{7}$ P. Carrier, S.-H. Wei, S. B. Zhang, and S. Kurtz, Phys. Rev. B 71, 165212 (2005).

${ }^{8}$ N. Miyashita, Y. Shimizu, N. Kobayashi, Y. Okada, and M. Yamaguchi, Proceedings of the 4th World Conference on Photovoltaic Energy Conversion, Hawaii (IEEE, Omnipress, 2006), p. 869.

${ }^{9}$ S. Fahy and E. P. O'Reilly, Appl. Phys. Lett. 83, 3731 (2003).

${ }^{10}$ S. R. Kurtz, A. A. Allerman, C. H. Seager, R. M. Sieg, and E. D. Jones, Appl. Phys. Lett. 77, 400 (2000).

${ }^{11}$ T. Suzuki, T. Yamaguchi, A. Yamamoto, and A. Hashimoto, Phys. Status Solidi C 0, 2769 (2003).

${ }^{12}$ A. Ohmae, N. Matsumoto, and Y. Okada, J. Cryst. Growth 251, 412 (2003).
${ }^{13}$ Y. Shimizu, N. Kobayashi, A. Uedono, and Y. Okada, J. Cryst. Growth 278, 553 (2005).

${ }^{14}$ S. R. Kurtz, J. F. Klem, A. A. Allerman, R. M. Sieg, C. H. Seager, and E. D. Jones, Appl. Phys. Lett. 80, 1379 (2002).

${ }^{15}$ A. Hashimoto, T. Yamaguchi, T. Suzuki, and A. Yamamoto, J. Cryst. Growth 278, 532 (2005).

${ }^{16}$ M. P. Vaughan and B. K. Ridley, Phys. Rev. B 72, 075211 (2005).

${ }^{17}$ C. M. Wolfe, G. E. Stillman, and W. T. Lindley, J. Appl. Phys. 41, 3088 (1970).

${ }^{18}$ A. K. Saxena, Phys. Rev. B 24, 3295 (1981).

${ }^{19}$ C. Erginsoy, Phys. Rev. 79, 1013 (1950).

${ }^{20}$ J. Li, P. Carrier, and S.-H. Wei, Phys. Rev. Lett. 96, 035505 (2006).

${ }^{21}$ K. M. Yu, W. Walukiewicz, J. Wu, D. E. Mars, D. R. Chamberlin, M. A. Scarpulla, O. D. Dubon, and J. F. Geisz, Nat. Mater. 1, 185 (2002).

${ }^{22}$ K.M. Yu, W. Walukiewicz, J. Wu, D.E. Mars, M.A. Scarpulla, O.D. Dubon, M.C. Ridgway, J.F. Geisz, in Semiconductor Defect Engineering: Materials, Synthetic Structures and Devices, Materials Research Society Symposia Proceedings No. 864, edited by S. Ashok, J. Chevallier, B. L. Sopori, M. Tabe, and P. Kiesel (MRS, Warrendale, PA, 2005), p. E8.1.1. ${ }^{23}$ A. Janotti, S. H. Wei, S. B. Zhang, and S. R. Kurtz, Phys. Rev. B 67, $161201(2003)$ 